\title{
On the shape of continuous wave infrared stimulated luminescence signals from feldspars: A case study
}

Pagonis, V.; Jain, Mayank; Thomsen, Kristina Jørkov; Murray, Andrew S.

Published in:

Journal of Luminescence

Link to article, DOI:

10.1016/j.jlumin.2014.03.010

Publication date:

2014

Document Version

Peer reviewed version

Link back to DTU Orbit

Citation (APA):

Pagonis, V., Jain, M., Thomsen, K. J., \& Murray, A. S. (2014). On the shape of continuous wave infrared stimulated luminescence signals from feldspars: A case study. Journal of Luminescence, 153, 96-103. https://doi.org/10.1016/j.jlumin.2014.03.010

\section{General rights}

Copyright and moral rights for the publications made accessible in the public portal are retained by the authors and/or other copyright owners and it is a condition of accessing publications that users recognise and abide by the legal requirements associated with these rights.

- Users may download and print one copy of any publication from the public portal for the purpose of private study or research.

- You may not further distribute the material or use it for any profit-making activity or commercial gain

- You may freely distribute the URL identifying the publication in the public portal 


\section{TITLE PAGE}

TITLE:

ON THE SHAPE OF CONTINUOUS WAVE INFRARED STIMULATED LUMINESCENCE SIGNALS FROM FELDSPARS: A CASE STUDY

\section{Author names and affiliations}

V. Pagonis ${ }^{\mathrm{a}, *}$, M. Jain ${ }^{\mathrm{b}}$, K. Thomsen ${ }^{\mathrm{b}}$ and A.S. Murray ${ }^{\mathrm{c}}$

${ }^{a}$ McDaniel College, Physics Department, Westminster, MD 21157, USA

${ }^{\text {b} R a d i a t i o n ~ R e s e a r c h ~ D e p a r t m e n t, ~ R i s ø ~ N a t i o n a l ~ L a b o r a t o r y ~ f o r ~ S u s t a i n a b l e ~ E n e r g y, ~ D a n i s h ~}$ Technical University, Frederiksborgvej 399, DK-4000 Roskilde, Denmark

cNordic Laboratory for Luminescence Dating, Department of Earth Sciences, Aarhus University, Risø DTU, DK-4000 Roskilde, Denmark

\section{* Corresponding author}

Vasilis Pagonis

Email: vpagonis@mcdaniel.edu

Phone: (410)-857-2481 Fax: (410)-386-4624 


\title{
ON THE SHAPE OF CONTINUOUS WAVE INFRARED STIMULATED LUMINESCENCE SIGNALS FROM FELDSPARS: A CASE STUDY
}

\author{
V. Pagonis ${ }^{\mathrm{a},{ }^{*}}$, M. Jain ${ }^{\mathrm{b}}$, K. Thomsen ${ }^{\mathrm{b}}$ and A.S. Murray ${ }^{\mathrm{c}}$
}

${ }^{a}$ McDaniel College, Physics Department, Westminster, MD 21157, USA

${ }^{b}$ Radiation Research Department, Risø National Laboratory for Sustainable Energy, Danish Technical University, Frederiksborgvej 399, DK-4000 Roskilde, Denmark

'Nordic Laboratory for Luminescence Dating, Department of Earth Sciences, Aarhus University, Risø DTU, DK-4000 Roskilde, Denmark

\begin{abstract}
The continuous-wave IRSL signals from feldspars (CW-IRSL) are known to decay in a nonexponential manner, and their exact mathematical description is of great importance in dosimetric and dating studies. This paper investigates the possibility of fitting experimental CWIRSL curves from a variety of feldspar samples, by using an analytical equation derived within the framework of a new model based on localized electronic recombinations of donor-acceptor pairs. 24 different types of feldspars were studied and their CW-IRSL signals are analyzed in order to establish the range and precision of numerical values for the fitting parameters in the analytical equation. The study searches for systematic trends in the fitting parameters, and for possible systematic differences between $\mathrm{K}$ and $\mathrm{Na}$ rich extracts from the same feldspar samples. Furthermore, results are compared from natural samples, freshly irradiated samples, and samples which had undergone anomalous fading. The results of this analysis establish broad numerical ranges for the fitting parameters in the model. Specifically the possible range of the dimensionless density $\rho^{\prime}$ was found to be $\rho^{\prime} \sim 0.002-0.01$. These experimentally established ranges of $\rho^{\prime}$ will help guide future modeling work on luminescence processes in feldspars. Small statistical differences were found between K-rich and Na-rich fractions of the same sample. However, the experimental data shows that the parameters depend on the irradiation dose, but do not depend on the time elapsed after the end of the irradiation process. All samples exhibited the power law of decay, with the range of the power law coefficient $\beta=0.6 \sim 1.1$.

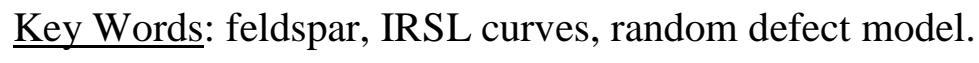


*Corresponding author. Email: vpagonis@mcdaniel.edu

Phone: (410)-857-2481 Fax: (410)-386-4624

\section{INTRODUCTION}

During the past twenty years there has been considerable experimental and modeling work trying to understand the nature and properties of luminescence signals from feldspars, especially in connection with the associated phenomenon of "anomalous fading” based on quantum mechanical tunneling (see for example the books [1]-[3]). The continuous-wave IRSL signals (CW-IRSL, also known as "infrared shine down” curves) from these materials are known to decay in a non-exponential manner and their origin as well as their exact mathematical description are an open research question. Mathematical and physical characterization of the shape of these CW-IRSL signals in feldspars is of great importance in dosimetric and dating studies (refs. [4]-[32]).

There is an abundance of experimental data as well as significant modeling work in the literature which suggests that the dominant process in anomalous fading in feldspars is tunneling from the ground state or via the excited state, or from both. Poolton et al., ([7-8],[17]]) explained IRSL in feldspars using a donor-acceptor model, in which electron tunneling occurs from the excited state and the band tail states of the IRSL trap at about $1.4 \mathrm{eV}$. Thomsen et al. [22-23] suggested that in this model, the beginning of the IRSL decay curve originates with the luminescence emitted from close donor-acceptor pairs, while the end of the IRSL curve most likely represents the tunneling of distant pairs. The recent experimental work by Poolton et al., [17] , Jain and Ankjærgaard [18], Ankjærgaard et al. [19], Andersen et al. [31] and Kars et al. [32] provided valuable information on the origin of these CW-IIRSL signals and support the presence of several competing mechanisms during the luminescence process. These mechanisms involve the tunneling processes in localized recombinations taking place from the ground and excited state of the trap, as well as charge migration through the conduction band-tail states.

There have also been significant developments in the modeling aspects of the luminescence mechanism for CW-IRSL signals, including the experimentally observed empirical power law of decay in feldspars ([14]-[16]). Kars et al. [11] and Larsen et al. [12] presented new types of models involving localized tunneling transitions, while Pagonis et al. [20] presented a new empirical kinetic model, in an attempt to describe tunneling via the excited state in 
feldspars. In an important development in this research area, Jain et al. [21] presented a new general kinetic model which quantifies localized electronic recombination of donor-acceptor pairs in luminescent materials. Recombination is assumed to take place via the excited state of the donor, and to take place between nearest-neighbors within a random distribution of centers. Two versions of the model were presented, an exact model that evolves in both space and time, and an approximate semi-analytical model evolving only in time. These authors found good agreement between the two models, and simulated successfully both thermally stimulated luminescence (TL) and optically stimulated luminescence (OSL). The model also demonstrated the power law behavior for OSL signals simulated within the model.

Pagonis et al. [33] examined the full model by Jain et al. [21], and obtained analytical expressions for the distribution of remaining donors at any time $t$ during the following experimental situations: TL, OSL, linearly modulated OSL and infrared stimulated luminescence (LM-OSL, LM-IRSL) and isothermal TL (ITL). These authors gave examples for the derived distributions of donors in each experimental case, and similarities and differences between the different experimental modes of stimulation were pointed out. They also demonstrates how LMIRSL signals in feldspars can be analyzed using the model, and what physical information can be extracted from such experimental data. Their analytical equations were tested by fitting successfully a series of experimental LM-IRSL data for Na- and K-feldspar samples available in the literature.

Kitis and Pagonis [34] showed that the system of simultaneous differential equations developed by Jain et al. [21] can be approximated to a very good precision by a single differential equation describing stimulated luminescence emission in this system. These authors were able to obtain analytical solutions of this single differential equation for several possible modes of stimulation, namely TL, OSL, LM-OSL and ITL. They also derived the exact analytical form for the power law behavior in this system, and demonstrated how typical experimental TL glow curves and CW-IRSL signals can be analyzed using the derived analytical equations, and what physical information that can be extracted from such experimental data.

The goals of the present paper are:

(a) To investigate the possibility of fitting experimental CW-IRSL curves from a variety of feldspar samples by using the analytical equation derived by Kitis and Pagonis [34] within the framework of the model by Jain et al. [21]. 
(b) To investigate the range of numerical values, as well as the precision of the kinetic parameters derived from fitting experimental data to the analytical equation derived by Kitis and Pagonis [34]. Special attention is paid in looking for systematic trends in the fitting parameters, and for possible systematic differences between $\mathrm{K}$ and $\mathrm{Na}$ rich extracts from the same feldspar samples.

(c) To compare the fitting parameters obtained from CW-IRSL curves measured in 3 types of samples: natural samples, freshly irradiated samples, and samples which have undergone anomalous fading. The specific goal here is to search for possible effects of laboratory irradiation and anomalous fading on the shape of the CW-IRSL signals.

(d) To examine the power law decay of these signals from different types of feldspars.

\section{ANALYTICAL SOLUTION FOR CW-IRSL SIGNALS WITHIN THE MODEL OF} JAIN ET AL. [21]

In this section we summarize briefly the main physical assumptions and equations used in the model of Jain et al. [21], and discuss the analytical equation used in this paper to fit experimental CW-IRSL curves. The main physical assumption in the model of Jain et al. [21] is the presence of a random distribution of hole traps in the luminescent volume, and an associated range of random nearest-neighbor recombination probabilities. Within the model, stimulated recombination takes place only via the excited state of the electron trap, by either optical or thermal stimulation. The concentration of holes is assumed to be much larger than the concentration of electron traps, and an electron can tunnel only to its nearest hole. In the exact form of the model, one writes a system of differential equations describing the traffic of electrons between the ground state, the excited state and the recombination center. These coupled differential equations contain two variables, namely the distance $r^{\prime}$ between donor-acceptor pairs and the time $t$.

Jain et al. [21] developed also an approximate semi-analytical model to describe the behavior of the system. This second version of the model evolves only in time, and the approximation used is based on introducing a critical tunneling lifetime $\tau_{c}$. The equations in the approximate semi-analytical model version of the model and for the case of CW-IRSL experiments are [21]: 


$$
\begin{aligned}
& \frac{d n_{g}}{d t}=-A n_{g}+B n_{e}, \\
& \frac{d n_{e}}{d t}=A n_{g}-B n_{e}-\frac{3 n_{e} \rho^{1 / 3}}{\tau_{c}}\left(\ln \frac{n_{o}}{n}\right)^{2 / 3} Z, \\
& L(t)=-\frac{d m}{d t}=\frac{3 n_{e} \rho^{1 / 3}}{\tau_{c}}\left(\ln \frac{n_{o}}{n}\right)^{2 / 3} z, \\
& \tau_{c}=s^{-1} \exp \left[\left(\frac{1}{\rho^{\prime}} \ln \frac{n_{o}}{n}\right)^{1 / 3}\right] .
\end{aligned}
$$

The following parameters and symbols are used in these expressions: $n_{g}$ and $n_{e}$ are the instantaneous concentrations of electrons in the ground state and in the excited state correspondingly. $m$ is the instantaneous concentration of acceptors (holes), $n$ is the instantaneous concentration of all the donors, and $N$ represents the instantaneous concentration of electrons in thermally disconnected states, such that $m=n+N=\left(n_{g}+n_{e}\right)+N$. The parameter $A$ represents the excitation rate from the ground to the excited state, and is equal to $A=\sigma(\lambda) I$ for the case of optical excitation. Here $\lambda$ is the optical stimulation wavelength, $\sigma(\lambda)$ is the optical absorption cross-section and $I$ is the light intensity $\left(\mathrm{cm}^{-2} \mathrm{~s}^{-1}\right)$. Additional parameters are the dimensionless number density of acceptors $\rho^{\prime}$, the critical tunneling lifetime $\tau_{c}$, the thermal excitation frequency factor $s$, and $z=1.8$ a dimensionless constant introduced in the model. $B$ is the relaxation rate from the excited into the ground state, and $L(t)$ is the instantaneous tunneling luminescence from recombination via the excited state. Under the detailed balance principle one also has $B=s$. Perhaps the most important physical parameter in the model is the dimensionless number density of acceptors $\rho^{\prime}$ and the critical tunneling lifetime $\tau_{c}$ which depends on the instantaneous concentration of donors $n$ as shown in equation (4). Jain et al. [29] simulated successfully both TL and optically stimulated luminescence (OSL) processes in their model, and also demonstrated the power law behavior for simulated OSL signals within the model. However, their approximate semi-analytical model was found to disagree with the exact solution of the model in the case of low values of the optical excitation probabilities $A=1-10 \mathrm{~s}^{-1}$ (Ref. [21], their figure 7a and 7b).

Kitis and Pagonis [34] showed that under certain simplifying physical assumptions, the 
system of equations (1)-(4) can be replaced accurately with a single differential equation, and they obtained the following analytical solution for the CW-IRSL luminescence intensity $L(t)$ at time $t$ :

$$
L(t)=3 n_{o} \rho^{\prime} F(t)^{2} z A e^{-F(t)} e^{-\rho^{\prime}[F(t)]^{3}},
$$

with the quantity $F(t)$ defined by:

$$
F(t)=\ln \left(1+z \int_{0}^{t} A d t^{\prime}\right)=\ln (1+z A t) .
$$

By substituting equation (6) into (5), the following analytical equation is obtained:

$$
L(t)=\frac{C[\ln (1+\mathrm{zA} t)]^{2} e^{-\rho^{\prime}[\ln (1+\mathrm{zA} t)]^{3}}}{1+\mathrm{zA} t},
$$

where $\mathrm{C}$ is a constant amplitude which depends on the experimental conditions. Equation (7) is used to fit the experimental CW-IRSL curves in this paper. The three variable fitting parameters are $C, \rho^{\prime}, A$.

\section{ANALYSIS OF IRSL CURVES FROM A SUITE OF N=24 FELDSPAR SAMPLES}

A first experimental study was carried out to investigate the possibility of fitting CWIRSL curves from a variety of feldspar samples by using the analytical equation (7).

It is to be noted that the equation 7 is developed for a full distribution of nearest neighbour distances. In a natural or a lab irradiated sample that has undergone preheating, it is exptected to have a truncated distribution since the pairs with a small lifetime will have recombined because of thermal excitations. Nonetheless for a preliminary investigations it is convenient to directly use eq 7 for fitting, rather than doing multiple simulations first for preheat and then for OSL. Since the form of the majority of data in the OSL curve is power law (Jain et al. 2012; Pagonis et al ??). At the moment it is not clear what is the overall error induced by such a simplification; it may change the absolute value of the model parameters. However, since all are investigations in this contribution are done in a relative mode (comparison across different doses, or delay times, or different samples), we think this issue is not significant here.

The suite of museum feldspar samples listed in Table 1 is chosen to represent a wide variety of samples, and also because it contains pairs of Na- and K-rich fractions extracted from the same feldspar samples (referred to as NF and KF in the rest of this paper and in some of the 
Figures). The main goal of this experiment is to establish the range and precision of the numerical values for the fitting parameters $C, \rho^{\prime}, A$. A secondary goal is to search for any systematic differences in the fitting parameters from natural and laboratory irradiated samples, as well as searching for possible differences between the available pairs of Kand Na- rich fractions.

The experimental sequence is as follows: a natural sample is preheated for $60 \mathrm{~s}$ at $320^{\circ} \mathrm{C}$, followed by measurement of the CW-IRSL signal by optical stimulation at $50^{\circ} \mathrm{C}$ for $500 \mathrm{~s}$ at $90 \%$ of full optical power (Mayank, is it necessary to add more experimental details here?). Immediately afterwards the sample undergoes CW-IRSL stimulation for $100 \mathrm{~s}$ at an elevated temperature of $325^{\circ} \mathrm{C}$, in order to empty the dosimetric trap. Subsequently the same aliquot is irradiated for $300 \mathrm{~s}$ (with what dose rate?) and the complete sequence is repeated to measure the CW-IRSL signal for the irradiated aliquot. The complete experimental sequence is then repeated for the rest of the $\mathrm{N}=24$ samples/aliquots shown in Table 1.

Figure 1a shows the result of fitting a typical CW-IRSL curve from the first two entries in Table 1. Two fitted curves are shown for a K-rich and Na-rich fraction (solid and open circles correspondingly). Only the first $100 \mathrm{~s}$ of the fitted signal are shown for clarity. The inset to Figure 1a shows the complete experimental data extending to $500 \mathrm{~s}$, on a log-log scale. The data points were measured $0.5 \mathrm{~s}$ apart, for a total of 1,000 data points collected along the CW-IRSL curve. The best fitting values of the three parameters in equation (7) are $C=6.8 \times 10^{5} \mathrm{cnts} / 0.5 \mathrm{~s}$, $\rho^{\prime}=0.0063$ and $A=4.30 \mathrm{~s}^{-1}$ for the K-rich fraction, and $C=8.9 \times 10^{5} \mathrm{cnts} / 0.5 \mathrm{~s}, \rho^{\prime}=0.0052$ and $A=4.10 \mathrm{~s}^{-1}$ for the Na-rich fraction of the feldspar sample. The residuals of the fitting procedure representing the difference between the experimental data and the corresponding best fit values are shown below the fitted graph for the K-rich sample, and are seen to be better than $\sim 5 \%$ of the corresponding CW-IRSL intensity for all points along the CW-IRSL signal. Typical $\mathrm{R}^{2}$ values for these fits are $\mathrm{R}^{2}=0.998$ indicating very good fits to the data. Figure $1 \mathrm{~b}$ shows the distribution of the residuals fitted to a Gaussian function centered at zero; despite a somewhat less satisfactory fit to the beginning of the data, an overall good global fit indicates that the residuals can be considered a reasonable approximation to white noise. Partly this systematic deviation at the beginning of the curve may arise from working with truncated nearest distributions rather than full distributions as assumed in the derivation of eq 7. 
Figures 2 and 3 show the results of analyzing N=24 experimental CW-IRSL curves similar to the one shown in Figure 1, obtained using the 24 feldspar samples in Table 1. Figure 2a shows the results for the dimensionless density $\rho^{\prime}$, for both natural samples (filled circles) and for laboratory irradiated samples (open circles). The average values of $\rho^{\prime}$ were $\rho^{\prime}=0.0037 \pm 0.0017(1 \sigma)$ for natural and $\rho^{\prime}=0.0050 \pm 0.0012(1 \sigma)$ for the laboratory irradiated samples correspondingly. These results indicate that the $(1 \sigma)$ precision of the extracted fitting parameters $\rho^{\prime}$ is of the order of $\sim 24 \%$ for laboratory irradiated samples and $\sim 46 \%$ for natural samples. The error bars shown at the left hand side of Figure 2a indicate the size of the standard deviation of the data, and the horizontal dashed and solid lines indicate the positions of the average values. Figure $2 \mathrm{~b}$ shows the same data as in Figure $2 \mathrm{a}$ in the form of histogram distributions. Inspection of the average values and standard deviations of the data in Figure 2ab shows that, even though some systematic differences may exist between the $\rho^{\prime}$ values of the natural and irradiated samples, the difference between the two sample populations is not statistically significant.

Figure 3a shows the best fitting values for the optical excitation probability of the $\mathrm{N}=24$ samples, with average values of $A=5.8 \pm 2.3\left(\mathrm{~s}^{-1}\right)(1 \sigma)$ and $A=6.1 \pm 2.2\left(\mathrm{~s}^{-1}\right)(1 \sigma)$ for the natural and irradiated samples correspondingly. These results indicate that the precision of the extracted fitting parameter $A$ is of the order of $\sim 36 \%$ for laboratory irradiated samples and $\sim 40 \%$ for natural samples. Clearly the data indicates that there is no significant statistical difference between the $A$ values for natural and irradiated samples.

Figure $3 b$ shows the best fitting values for the constant $C$ in equation (7); this constant is proportional to the intensity of the CW-IRSL curve at time $t=0$. As may be expected, there is wide variation in the values of $\mathrm{C}$, which covers approximately 4 orders of magnitude from

$\mathrm{C} 100$ to $\mathrm{C} \sim 10^{6}$ (cts/0.5 s) This data indicates again that there are no apparent systematic differences between the $\mathrm{C}$ values for natural and irradiated samples.

\section{ANALYSIS OF CW-IRSL CURVES FROM THE WORK BY THOMSEN ET AL., [22]}

Thomsen et al. [22] determined fading rates for sedimentary feldspar samples using both different stimulation and detection windows. They investigated the fading rate for both the initial part and the final part of the luminescence signal, and found that stimulation at elevated 
temperatures reduces the apparent fading rate. These authors concluded that some luminescence signals from sedimentary feldspars fade at a much lower rate than conventional IRSL signals. In the same work these authors determined the g-values by carrying out a series of sensitivity corrected measurements ( $\mathrm{L}_{\mathrm{x}} / \mathrm{T}_{\mathrm{x}}$ measurements) using a modified SAR procedure (Murray and Wintle, [28]; Auclair et al., [29]). During these measurements, sensitivity changes are explicitly corrected by the use of a test dose $\left(T_{x}\right)$. Furthermore, a prompt measurement was undertaken after each delayed measurement, in order to correct for any further sensitivity changes occurring over time. Optical stimulation was carried out at $50^{\circ} \mathrm{C}$ for $100 \mathrm{~s}$, and a standard preheat of $60 \mathrm{~s}$ at $250^{\circ} \mathrm{C}$ followed each irradiation (Auclair et al., [29]). The delay times between the end of irradiation and measurement of the CW-IRSL signals varied from a few seconds for prompt measurements, to long delays up to $\sim 17$ hours.

This section presents the results of fitting a series of $L_{x}, T_{x}$ measurements carried out by Thomsen et al. [22]. However the current study is not concerned with the fading rates of the IRSL signal. Instead, the current analysis looks for systematic changes in the overall shape of the IRSL curve, by examining the behavior of the fitting parameters $\rho^{\prime}$ and $A$. The specific goal of the analysis is to show that one can fit accurately the $\mathrm{L}_{\mathrm{x}}$ and $\mathrm{T}_{\mathrm{x}} \mathrm{CW}$-IRSL curves using equation (7) from Kitis and Pagonis [34], and to investigate whether these signals can be characterized in a consistent manner. A secondary goal is to search for systematic differences in the fitting parameters from available pairs of K- and Na- rich fractions from the same feldspar samples.

Four feldspar samples were studied in detail. The first sample was a glaciofluvial feldspar from Russia (laboratory number 963806, grain size 106-212 $\mu \mathrm{m}$ ). The second sample was glaciofluvial feldspar from Greenland (laboratory code 951020, 212-300 $\mu \mathrm{m}$ ). Experimental CW-IRSL curves were analyzed from both K- rich and Na- rich extracts from each of these two samples, for a total of 4 samples studied.

The series of data been analyzed for each of these 4 samples consists of $12 L_{x}$ curves measured for each of 6 different aliquots of the same sample. This procedure yields a total of $\mathrm{N}=$ $N=12 \times 6=72$ IRSL curves for the K-rich extracts, and an equal number of N=72 IRSL curves for the Na-rich extracts. An additional $N=72 T_{x}$ curves were analyzed in each of these cases.

The $\mathrm{N}=72$ curves analyzed for each sample represent a mixture of freshly irradiated states (prompt measurements), and states which have undergone anomalous fading for various elapsed times after irradiation (up to $\sim 17 \mathrm{hrs}$ ). The specific goal here is to search for possible effects of 
laboratory irradiation and anomalous fading on the shape of the CW-IRSL signals. The results also provide an estimate of the reproducibility of the fitting parameters between different aliquots of the same sample, which can be considered an estimate of the precision of this type of fitting curve analysis.

A typical result of the best fitting parameters obtained fitting $\mathrm{N}=72 \mathrm{~L}_{\mathrm{x}}$ curves using equation (7) is shown in Figure 4a (open circles). Also shown in the same Figure 4a are N=72 data points obtained from analyzing the corresponding $\mathrm{T}_{\mathrm{x}}$ curves (open triangles). The sample analyzed in Figure 4 was the K-rich extract of sample number 963806. The aliquots were irradiated for $600 \mathrm{~s}$ before measuring the $\mathrm{L}_{\mathrm{x}}$ curves, and for $300 \mathrm{~s}$ before measurement of the $T_{\mathrm{x}}$ curves (Karen, at what dose rate?). The error bars shown at the right hand side of Figure 4a indicate the size of the standard deviation of the data $(1 \sigma)$, and the horizontal dashed and solid lines indicate the positions of the average values for the $\mathrm{L}_{\mathrm{x}}$ and $\mathrm{T}_{\mathrm{x}}$ curves.

Inspection of the data in Figure 4a shows that there is no systematic correlation of the values of $\rho^{\prime}$ with the time delay after the end of irradiation. This seems to indicate that the shape of the CW-IRSL curves does not change very significantly during the modified SAR procedure used by Thomsen et al. [22]. However, the value of $\rho^{\prime}$ for the lower dose aliquots (i.e. from the $\mathrm{T}_{\mathrm{x}}$ signal, triangle shapes in Figure 4a), are systematically lower than the corresponding $\rho^{\prime}$ values for the higher dosed aliquots (the $\mathrm{L}_{\mathrm{x}}$ signals, circle shapes). The average values of $\rho^{\prime}$ were $\rho^{\prime}=0.0063 \pm 0.0003(1 \sigma)$ for the $\mathrm{L}_{\mathrm{x}}$ signals, and $\rho^{\prime}=0.0057 \pm 0.0003(1 \sigma)$ for the $\mathrm{T}_{\mathrm{x}}$ signals. The histogram distribution of the same data is shown in Figure 4b and it indicates that the value of $\rho^{\prime}$ may depend on the irradiation dose, although the size of the error bars in Figure 4a shows two closely overlapping populations. The dependence of $\rho^{\prime}$ on the irradiation dose is an important experimental effect, which could have implications for both experimental work and for modeling. This presence of this effect was verified for the other samples in this study, although the effect seems to be stronger for some samples and weaker for others.

Figure 5a shows the corresponding results for the optical excitation probability $A$ extracted from the $T_{x}$ signals (open triangles) and the $L_{x}$ signals (open circles). The value of $A$ from the $T_{x}$ signal are slightly lower than the corresponding values from the $L_{x}$ signals, although the percent differences between them are rather small. The corresponding histogram distribution of the same data is shown in Figure $5 b$, and the average values were $A=6.6 \pm 0.2\left(s^{-1}\right)$ for the $L_{x}$ 
signals, and $A=6.8 \pm 0.2\left(s^{-1}\right)$ for the $\mathrm{T}_{\mathrm{x}}$ signals. No systematic differences for the $A$ values and no correlation of $A$ with the delay time were apparent, at least within the precision of the experiments described here.

Figure 6a shows a comparison of the $\rho^{\prime}$ values for the K-rich and Na-rich extracts of sample 963806. The data shown is for the $\mathrm{L}_{\mathrm{x}}$ curves (samples irradiated for $600 \mathrm{~s}$ ). Once more there is no apparent correlation of the values of $\rho^{\prime}$ with the delay time after the end of irradiation. Figure 6b shows a similar comparison for the corresponding values of the optical excitation probability A for the K-rich and Na-rich samples. The data in Figures 6ab seem to indicate no significant statistical differences between the two fractions of sample 963806, at least within the precision of the experiment.

Figures 7 present the same above comarison for the sample 951020. ;

7a shows results for the dimensionless charge density parameter $\rho^{\prime}$ for for the sodium fraction 951020NF for two given doses 400 and $200 \mathrm{~s}$ as a funciton of delay time in the fading experiment, $7 \mathrm{~b}$ the combined histograms of $\rho^{\prime}$ for all these delay times, and 7c the comparison of $\rho^{\prime}$ for the sodium and $\mathrm{K}$ rich fractions in the fading experiment. The aliquots were irradiated for $400 \mathrm{~s}$ before measuring the $\mathrm{L}_{\mathrm{x}}$ curves, and for $200 \mathrm{~s}$ before measurement of the $\mathrm{T}_{\mathrm{x}}$ curves (at what dose rate?). Once more the error bars in these figures indicate the size of the standard deviation of the data $(1 \sigma)$, and the horizontal dashed and solid lines indicate the average values of $\rho^{\prime}$ for the $\mathrm{L}_{\mathrm{x}}$ and $\mathrm{T}_{\mathrm{x}}$ curves. The data in Figure $7 \mathrm{~b}$ and provides additional evidence that the value of $\rho^{\prime}$ can possibly depend on the irradiation dose. The average values of $\rho^{\prime}$ were $\rho^{\prime}=0.0056 \pm 0.0002(1 \sigma)$ for the $\mathrm{L}_{\mathrm{x}}$ signals, and $\rho^{\prime}=0.0049 \pm 0.0002(1 \sigma)$ for the $\mathrm{T}_{\mathrm{x}}$ signals. Once more no systematic correlation of the values of $\rho^{\prime}$ with the time delay is found. Similarly the data in Figure 8a shown for the $\mathrm{L}_{\mathrm{x}}$ curves (samples irradiated for $400 \mathrm{~s}$ ) shows a significant statistical difference between $\rho^{\prime}$ for the two extracts of sample 963806, at least within the precision of the experiment.

Clearly further systematic experimental work is necessary, in order to verify the effect of irradiation dose on the fitting parameters and on the shape of the CW-IRSL curves. In addition, more experimental work on different types of feldspars is necessary to ascertain (or not) any significant differences between $\mathrm{K}$ - and $\mathrm{Na}$ - rich fractions. 
All of the CW-IRSL curves analyzed in this paper displayed the power law behavior at long stimulation times. For example, the data in the inset of Figure 1a show a linear behavior on a log-log scale, according to the power law behavior:

$\ln L(t)=c-\beta \ln t$

where $L(t)$ is the luminescence intensity, $t$ is the IR-simulation time and $\beta$ is the dimensionless power law coefficient. For example, the inset of Figure 1a shows two examples of the power law, in which the CW-IRSL intensity is linear on a log-log scale at long stimulation times.

Figure 8 shows an example of the distribution of the coefficient $\beta$ for the K-rich fraction of sample 963806 and for both the $L_{x}$ and $T_{x}$ curves (600s and 300s irradiation times correspondingly). Once more, the histogram distribution of the data indicates that the value of $\beta$ depends on the irradiation dose, although the two populations closely overlap. The values of $\beta$ are in agreement with previous experimental work by Bailiff and Poolton [24], Baril [16] and Bailiff and Barnett [27] who showed that the IRSL decay follows a power law type of decay. More generally, in some of these experimental studies the complete CW-IRSL signal was found to follow a Becquerel decay law of the form $A /\left(1+t / t_{0}\right)^{\beta}$ where $A, \beta$ and $t_{0}$ are constants. A comparison between equation (7) and the Becquerel decay law will be presented elsewhere.

\section{DISCUSSION AND CONCLUSIONS}

The results of this paper show that equation (7) can be used to fit accurately CW-IRSL signals from a variety of feldspar samples. The results establish broad numerical ranges for the fitting parameters $C, \rho^{\prime}, A$ in the model by Jain et al. [21] as applied to feldspars. Most notably, the range of the dimensionless density $\rho^{\prime}$ was found to be rather limited, from a low of $\sim 0.002$ to a maximum of $\sim 0.01$. These experimentally established ranges of $\rho^{\prime}$ will help guide future modeling work on luminescence processes in feldspars.

The results also showed that the values of the fitting parameters may be statistically different for K-rich and Na-rich fractions of the same feldspar sample, however a more systematic study of this effect is necessary to establish the validity of these differences. The experimental data presented here seems to support some systematic dependence of the fitting parameters on the irradiation dose; the systematic increase in $\rho^{\prime}$ for higher dose for all samples suggests an increase in the hole density with dose. The same effect has recently been 
documented by Morthekai et al. (2013) using fading experiments after radiations of different ionisation qualities. This phenomenon can have important consequences for future experimental and modeling work, especially with regards to fading correction. As expected, the time elapsed after the irradiation appears to have no significant statistical effect on the parameters $\rho^{\prime}$, A. All samples examined here exhibit the power law of decay, with the range of values of the power law coefficient $\beta=0.6 \sim 1.1$, in agreement with previous experimental studies.

Kitis and Pagonis [34] showed that the analytical Equation (7) also applies to isothermal signals described by the model of Jain et al. [21]. In the case of isothermal experiments and for signals derived from a single trap, it is not clear at this point whether the value of $\rho^{\prime}$ would be the same as the value derived from analysis of the CW-IRSL experiments. This is worth future investigations. A comparison of $\rho^{\prime}$ values for optical and thermal stimulation, as well as those estimated using laboratory fading experiments at the room temperature will throw further light on pathways used in these different recombination processes.

However, one would definitely expect the value of the stimulation probability A to strongly depend on the temperature used during the isothermal experiment. It is suggested that such a combined CW-IRSL and isothermal experiment on the same samples would provide valuable information on the luminescence processes in feldspars. 


\section{REFERENCES}

[1] Bøtter-Jensen L, McKeever S W S and Wintle A G 2003 Optically Stimulated Luminescence Dosimetry (Amsterdam: Elsevier)

[2] Chen R and McKeever S W S 1997 Theory of Thermoluminescence and Related Phenomena (Singapore: World Scientific)

[3] Chen, R. and Pagonis, V., 2011. Thermally and Optically Stimulated Luminescence: A Simulation Approach (Chichester: Wiley and Sons).

[4] Visocekas, R. Nucl. Tracks Radiat. Meas. 10, (1985) 521-529.

[5] Visocekas R, Spooner N A, Zink A and Blank P Radiat. Meas. 23 (1994) 371-85

[6] Duller, G.A.T. and Bøtter-Jensen, L. Radiat. Prot. Dosim. 47, (1993) 683-688.

[7] Poolton, N.R.J., Wallinga, J., Murray, A.S., Bulur, E., Bøtter-Jensen, L. Phys. Chem. Minerals 29, (2002) 210-216.

[8] Poolton, N.R.J., Ozanyan, K.B., Wallinga, J., Murray, A.S., Bøtter-Jensen, L. Phys. Chem. Minerals 29, (2002) 217-225.

[9] Li, B., Li S-H. Radiat. Meas. 46, (2010) 29-36.

[10] Li, B., Li S-H. J. Phys. D: Appl. Phys. 41, (2008) 225502 (15pp). doi:10.1088/00223727/41/22/225502.

[11] Kars, R. H., Wallinga, J., Cohen, K. M. Radiat Meas. 43, (2008) 786-90.

[12] Larsen, A., Greilich, S., Jain, M., Murray, A.S. Radiat. Meas. 44, (2009) 467-471. 
[13] Huntley, D.J., Lamothe, M. Canad. J. Earth Sci. 38, (2001) 1093-1106.

[14] Delbecq C J, Toyozawa Y and Yuster P H Phys. Rev. B 9 (1974) 4497-505

[15] Huntley D J J. Phys.: Condens. Matter 18 (2006) 1359-65

[16] Baril, M.R., 2002. Ph.D. Thesis, Simon Fraser University, Burnaby, BC, Canada. Available online at: www.cfht.hawaii.edu/ baril/Temp/baril_phdthesis.pdf

[17] Poolton N R J, Kars R H, Wallinga J , Bos A J J J. Phys.: Condens. Matter 21 (2009) 485505

[18] Jain M , Ankjærgaard C Radiat. Meas. 46 (2011) 292-309

[19] Ankjærgaard, C., Jain, M., Kalchgruber, R., Lapp, T., Klein, D., McKeever, S.W.S., Murray, A.S., Morthekai, P. Radiat Meas. 44, (2009) 576-581.

[20] Pagonis, V., Jain, M., Murray, A.S., Ankjærgaard, C. , Chen, R. Radiat. Meas., (2012) http://dx.doi.org/10.1016/j.radmeas.2012.02.012, in press.

[21] Jain, M , Guralnik, B , Andersen, M T. J. Phys.: Condens. Matter 24 (2012) 385402 (12pp)

[22] Thomsen, K.J., Murray, A.S., Jain, M., Bøtter-Jensen, L. Radiat Meas. 43, (2008) 14741486.

[23] Thomsen, K.J., Murray, A.S., Jain, M. Geochronometria, 38, (2011) 1-13.

[24] Bailiff I. K. , Poolton N. R. J. Nucl. Tracks Radiat. Meas. 18, (1991) 111-118.

[25] Murray, A.S., Buylaert, J.P., Thomsen, K.J., Jain, M. Radiat Meas. 44, (2009) 554-559. 
[26] Baril, M.R., Huntley, D. J. Journal of Physics: Condensed Matter 15, (2003) 8011-8027

[27] Bailiff, I.K., Barnett, S.M., Radiat. Meas. 23, (1994) 541-545.

[28] Murray, A.S., Wintle, A.G., Radiat. Meas. 32 (2000) 57-73.

[29] Auclair, M., Lamothe, M., Huot, S., Radiat. Meas. 37 (2003) 487-492.

[30] A. Molodkov, I. Jaek and V. Vasilchenko, Geochronometria 26 (2007), pp 11-17

DOI 10.2478/v10003-007-0007-0

[31] Andersen, M.T., Jain, M., Tidemand-Lichtenberg, P., 2012. Red-IR stimulated luminescence in K-feldspar: single or multiple trap origin? Journal of Applied Physics 112.

[32] Kars, R. H., Poolton, N.R.J., Jain, M., Ankjærgaard, C., Dorenbos, P. and Wallinga, J., (2013). Radiat. Meas. In Press. http://dx.doi.org/10.1016/j.radmeas.2013.05.002

[33] Pagonis, V., Phan, H., Ruth, D. and Kitis, G., Radiat. Meas, (2013), http://dx.doi.org/10.1016/j.radmeas.2013.08.006

[34] Kitis, G. and Pagonis, V. J. Lum., 137 (2013) 109-115. 


\section{LIST OF FIGURES}

\section{Figure 1}

(a)The result of fitting a typical CW-IRSL curve for a K-rich and Na-rich fraction of the same feldspar sample (solid and open circles correspondingly) using equation (7). Only the first $100 \mathrm{~s}$ of the fitted signal are shown for clarity. The inset shows the complete experimental data extending to $500 \mathrm{~s}$, on a log-log scale. The residuals of the fitting procedure are shown below the fitted graph for the K-rich sample

(b)The distribution of the residuals fitted to a Gaussian function centered at zero, indicating that the residuals can be considered a reasonable approximation to white noise.

\section{Figure 2}

The results of analyzing $\mathrm{N}=24$ experimental CW-IRSL curves from the 24 feldspar samples in Table 1 using equation (7) by Kitis and Pagonis (2013).

(a) The dimensionless density $\rho^{\prime}$, for both natural samples (filled circles) and for laboratory irradiated samples (open circles). The error bars shown at the left hand side of the figure indicate the size of the standard deviation of the data, and the horizontal dashed and solid lines indicate the positions of the average values.

(b)The same data as in (a) is shown in the form of histogram distributions.

\section{Figure 3}

(a)The best fitting values for the optical excitation probability of the $\mathrm{N}=24$ samples, for the natural and irradiated samples. This data does not support significant statistical differences between the $A$ values for natural and irradiated samples.

(b)The best fitting values for the constant $C$ in equation (7); there is wide variation in the values of $\mathrm{C}$ covering approximately 4 orders of magnitude from $\mathrm{C} \sim 100$ to $\mathrm{C} 10^{6}$ (cts/0.5s).

\section{Figure 4}

(a) Results of the best fitting $\rho^{\prime}$ parameters obtained fitting $\mathrm{N}=72 \mathrm{~L}_{\mathrm{x}}$ curves from Thomsen et al. [22] using equation (7) (open circles). The open triangles are $\mathrm{N}=72$ data points obtained 
from analyzing the corresponding $\mathrm{T}_{\mathrm{x}}$ curves (open triangles). The sample analyzed was the $\mathrm{K}$ rich extract of sample number 963806. The error bars shown at the right hand side of this figure indicate the size of the standard deviation of the data $(1 \sigma)$, and the horizontal dashed and solid lines indicate the average values.

(b) The same data as in (a) shown as a histogram distribution, that the value of $\rho^{\prime}$ may depend on the irradiation dose.

\section{Figure 5}

(a) The results for the optical excitation probability $A$ for the same sample as in Figure 4, with the $T_{x}$ signals shown as open triangles and the $L_{x}$ signals shown as open circles.

(b) The corresponding histogram distribution of the same data as in (a). No systematic differences for the $A$ values and no correlation of $A$ with the delay time are apparent.

\section{Figure 6}

(a) Comparison of the $\rho^{\prime}$ values for the K-rich and Na-rich extracts of sample 963806. The data shown is for the $\mathrm{L}_{\mathrm{x}}$ curves (samples irradiated for $600 \mathrm{~s}$ ).

(b) A similar comparison for the corresponding values of the optical excitation probability A for the K-rich and Na-rich fractions of the same sample.

\section{Figure 7}

(a) The results for the dimensionless charge density parameter $\rho^{\prime}$ for sample 951020. The aliquots were irradiated for $400 \mathrm{~s}$ before measuring the $\mathrm{L}_{\mathrm{x}}$ curves, and for $200 \mathrm{~s}$ before measurement of the $\mathrm{T}_{\mathrm{x}}$ curves. The histogram distribution of the same data as in (a)

\section{Figure 8}

(a) A comparison of the Avalues for the K-rich and Na-rich extracts of sample 963806. The data shown is for the $\mathrm{L}_{\mathrm{x}}$ curves (samples irradiated for $400 \mathrm{~s}$ ). No significant statistical difference between the two extracts of sample 963806 is found, at least within the precision of the experiment. 
(b) Example of the distribution of the coefficient $\beta$ for the K-rich fraction of sample 963806 and for both the $L_{x}$ curves and $T_{x}$ curves (600s and 300s irradiation times correspondingly). 
Figure 1
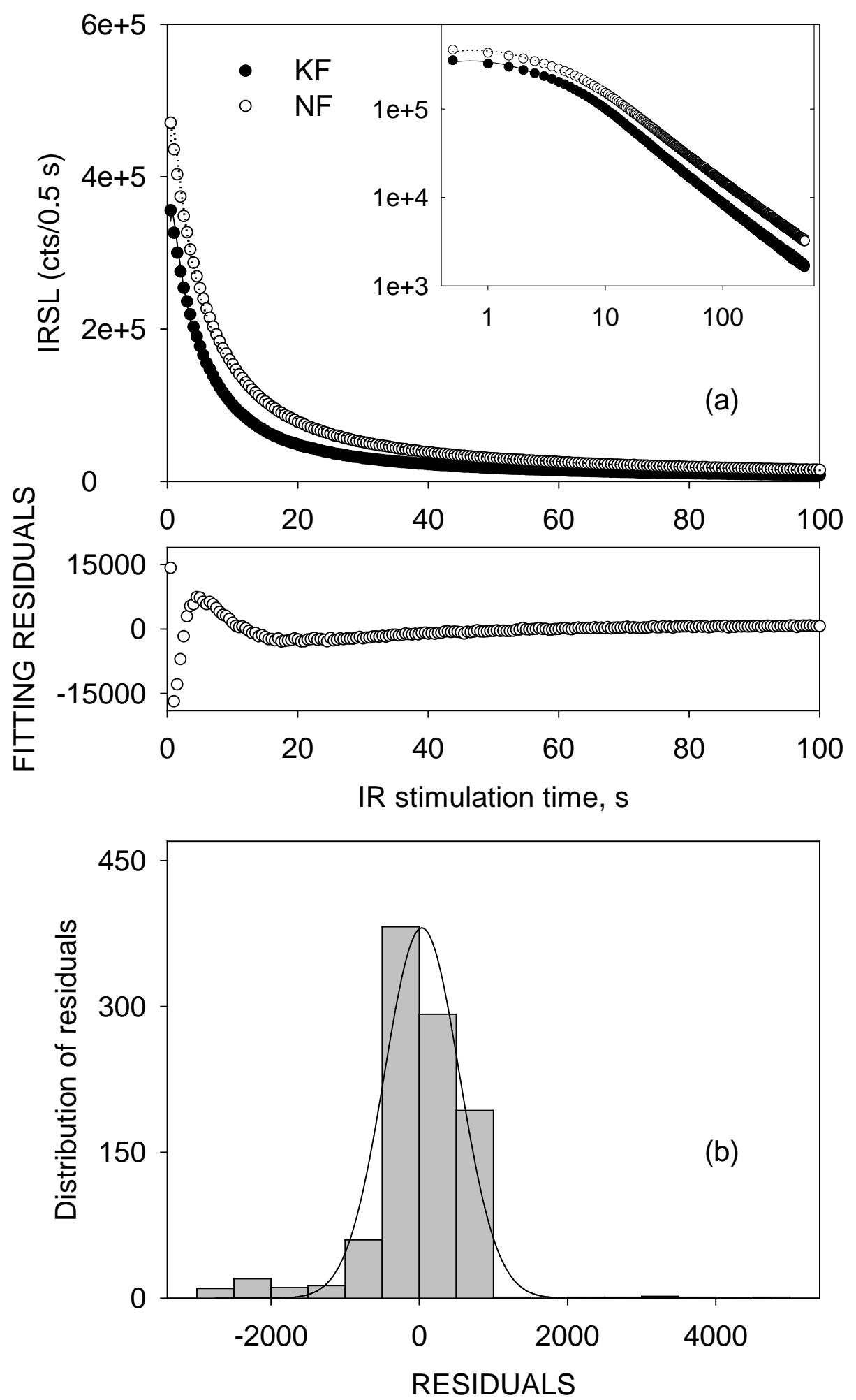
Figure 2
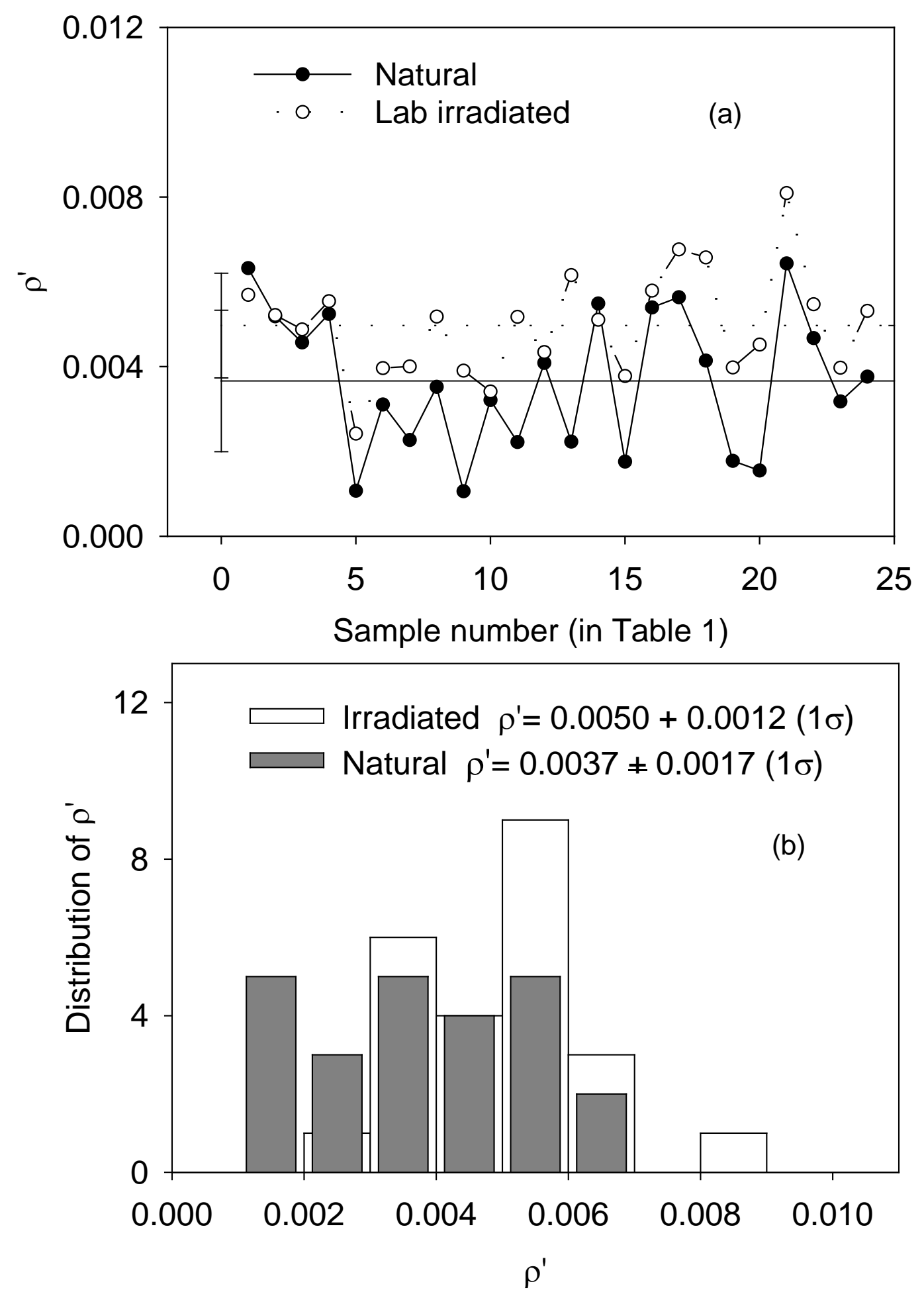
Figure 3

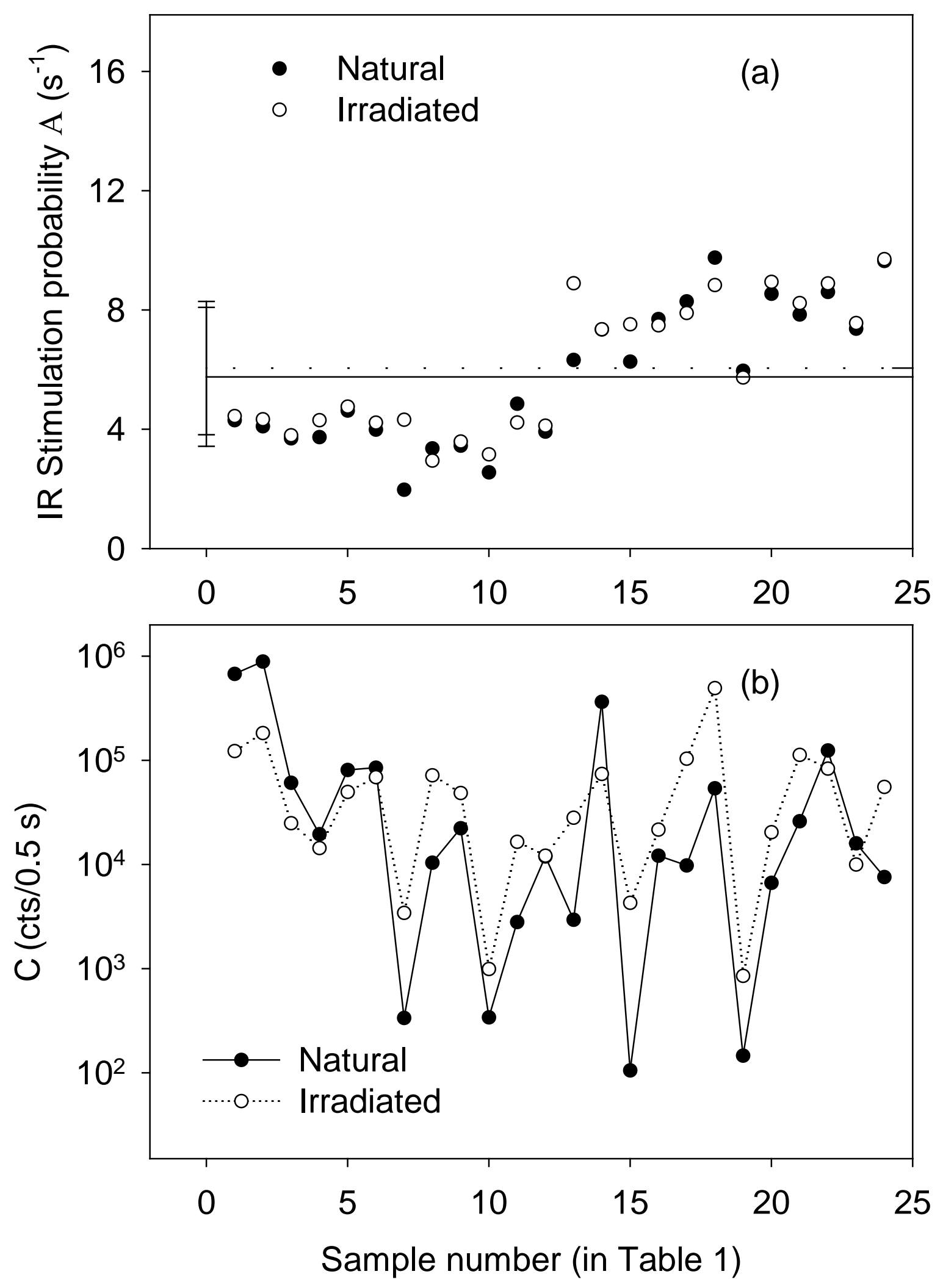



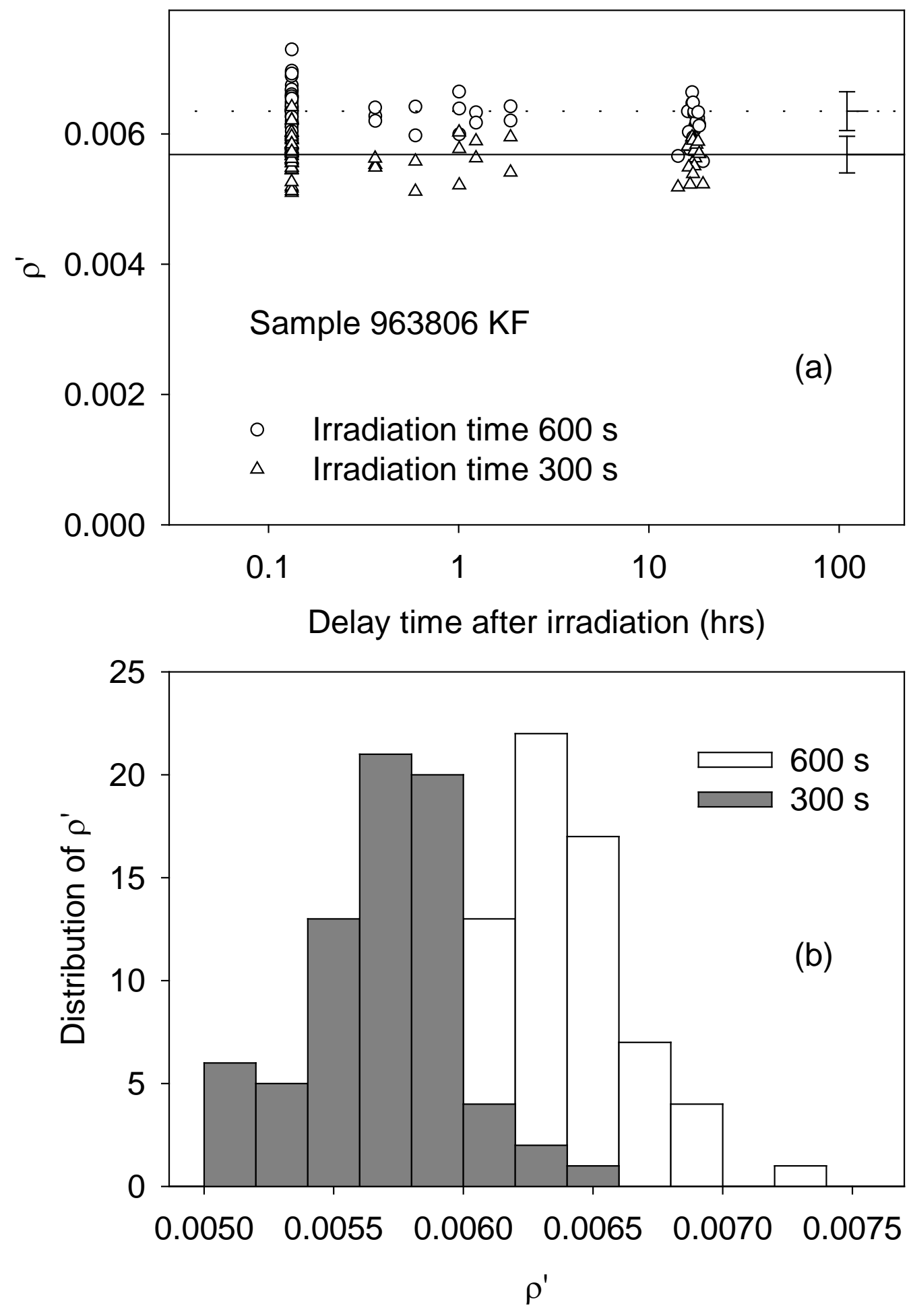

Figure 4 

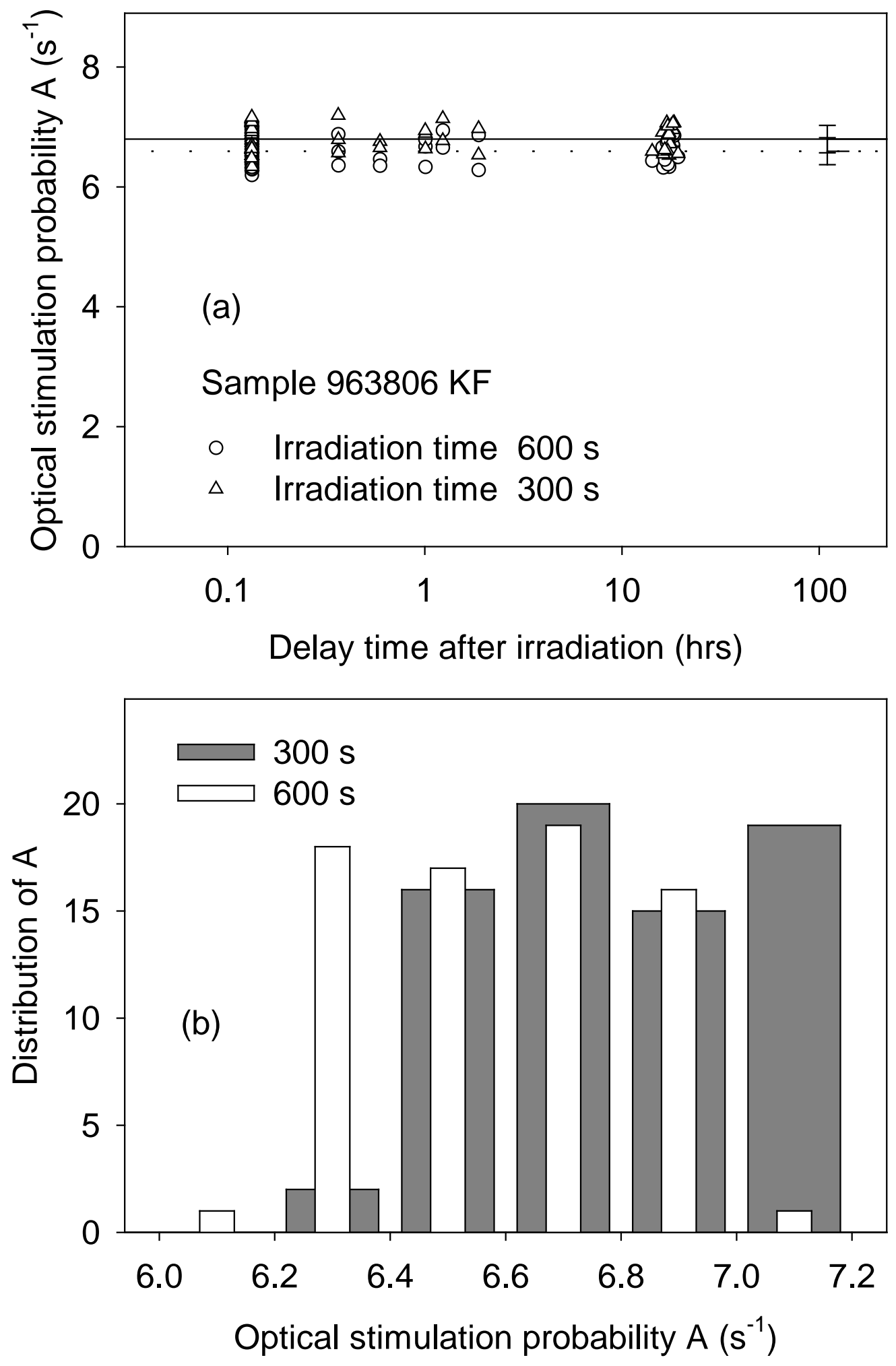

Figure 5 
Figure 6
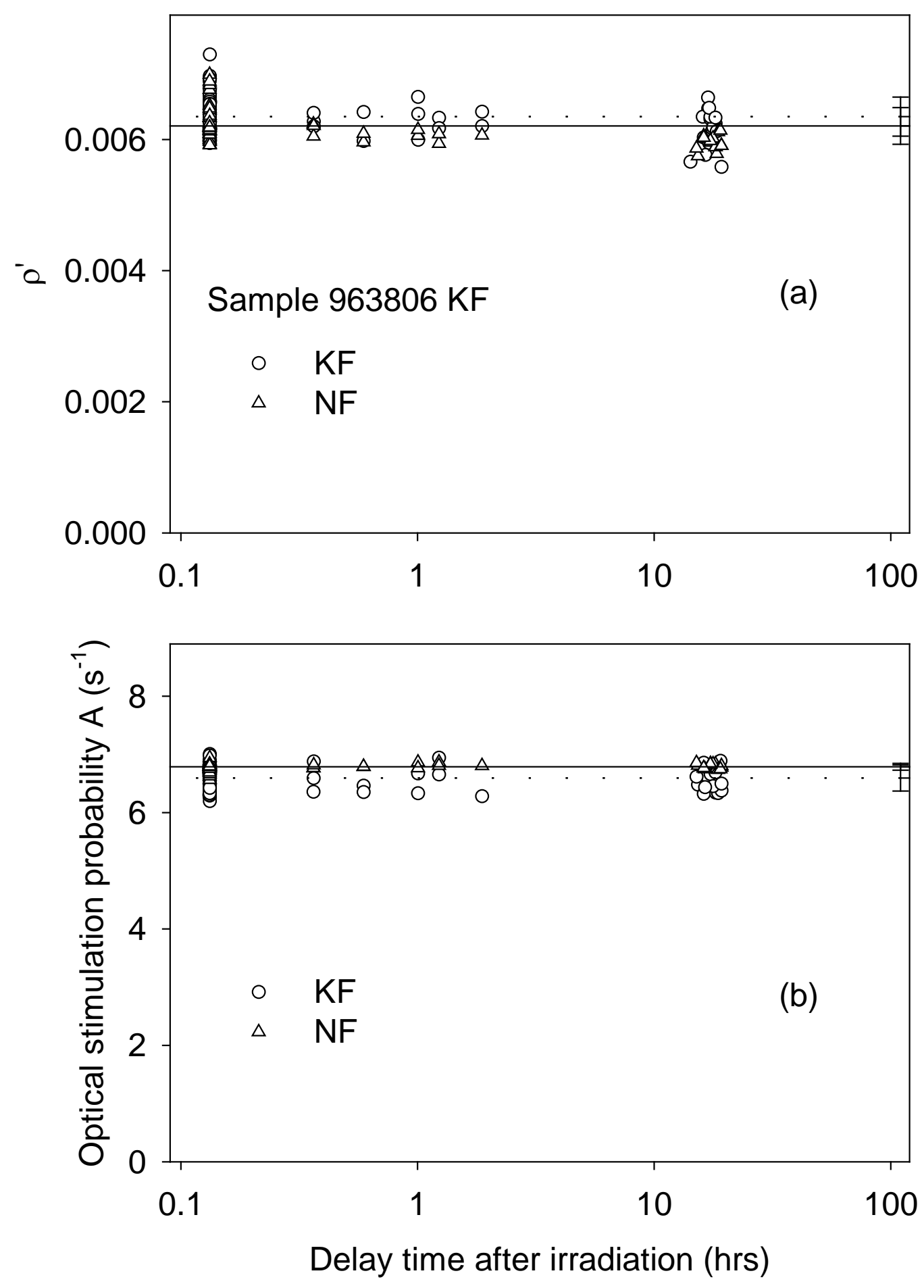
Figure 7
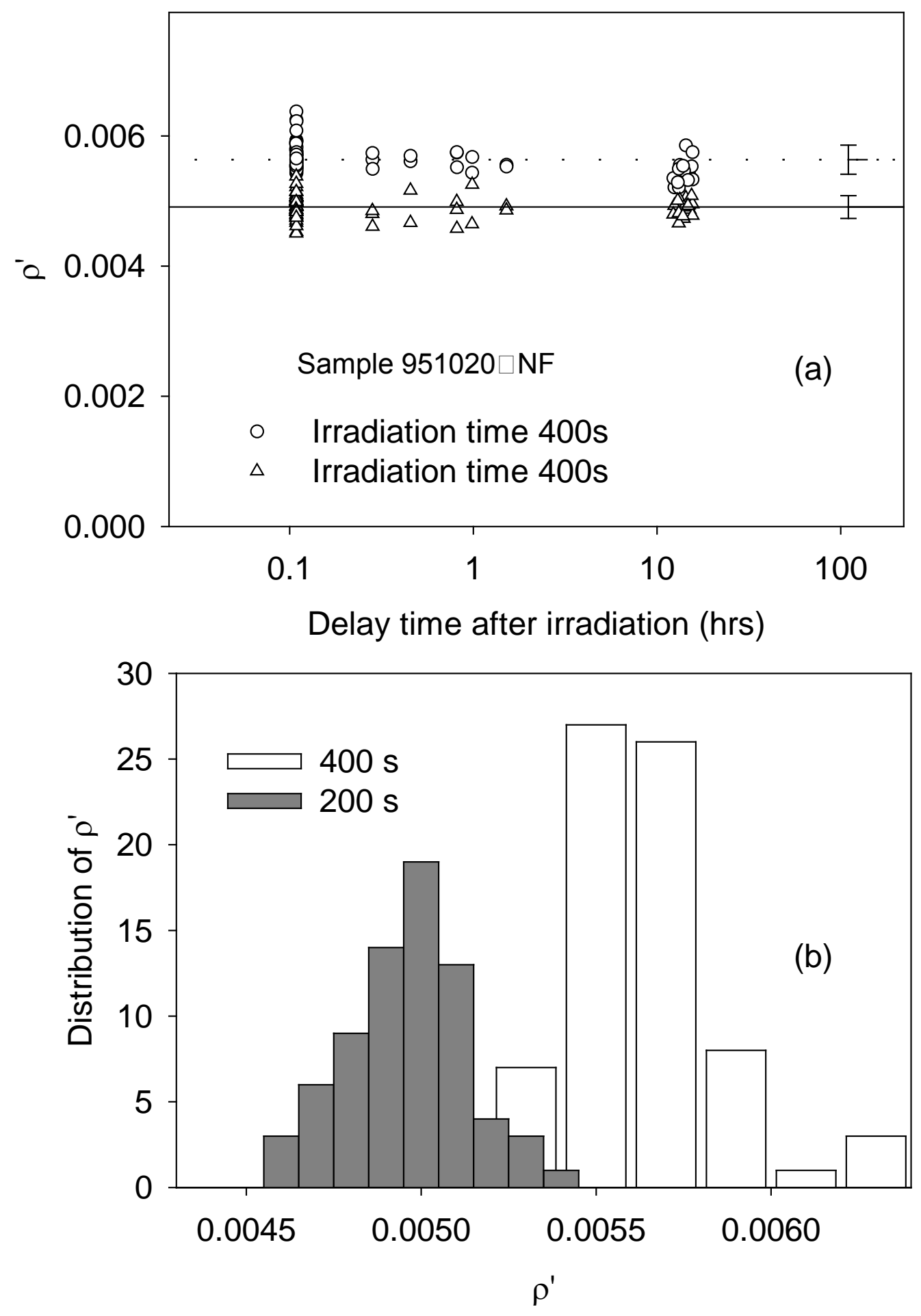

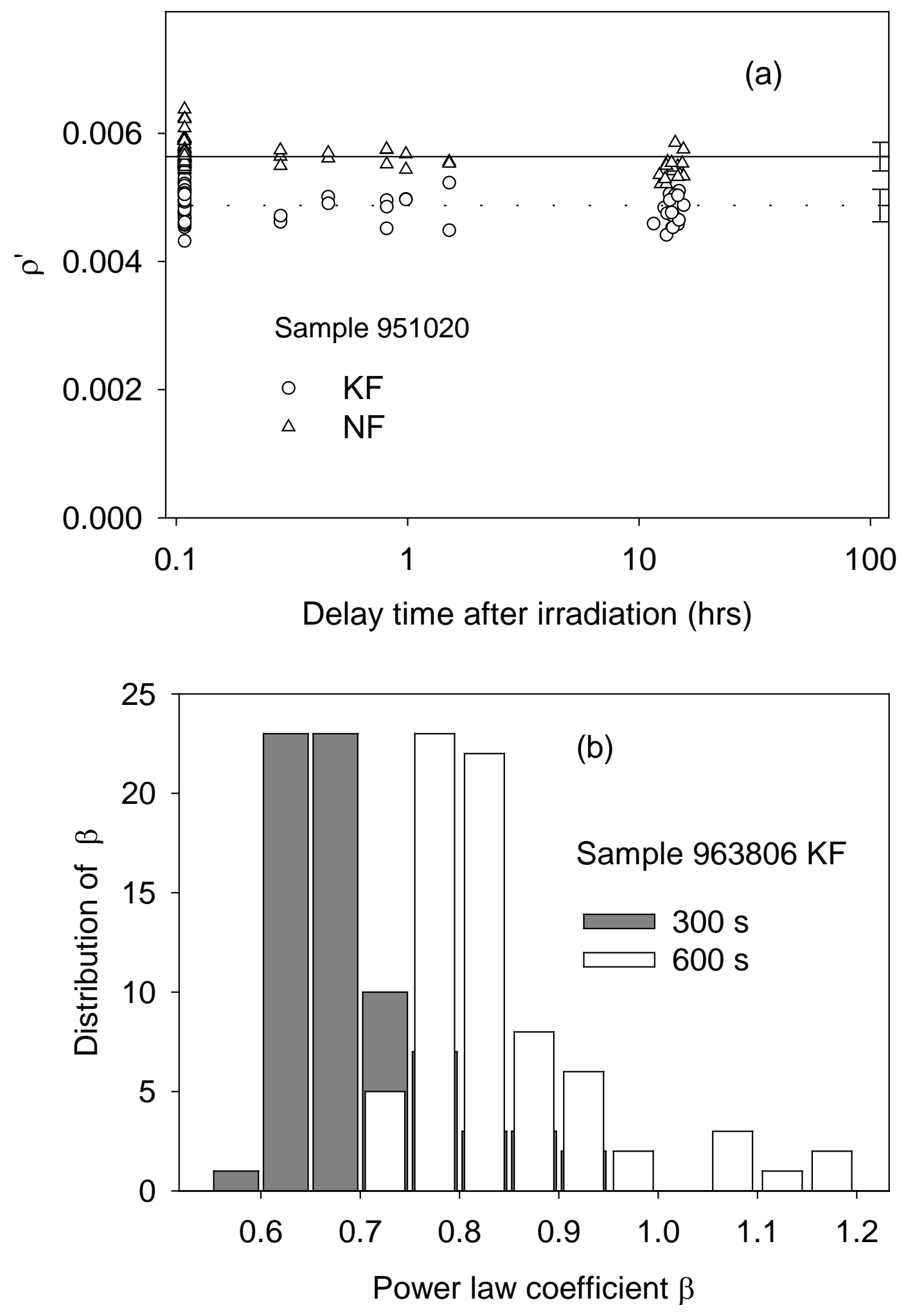

Figure 8 


\section{TABLE 1}

List of feldspar samples analyzed in this paper. KF and NF indicate K-rich and Na-rich fractions of the same sample.

\begin{tabular}{|c|c|c|c|}
\hline $\begin{array}{l}\text { Sample position } \\
\text { in disk }\end{array}$ & Sample no & sample lab code & FELDSPAR TYPE/NAME \\
\hline 1 & 1 & 981007 & FK \\
\hline 3 & 2 & 981007 & NF \\
\hline 5 & 3 & 075403 & FK \\
\hline 7 & 4 & 075403 & NF \\
\hline 9 & 5 & 092203 & FK \\
\hline 11 & 6 & 092203 & $\mathrm{NF}$ \\
\hline 13 & 7 & K-FELDSPAR & Spain \\
\hline 15 & 8 & Microcline & \\
\hline 17 & 9 & 970203 & $\mathrm{KF}$ \\
\hline 19 & 10 & 970203 & NF \\
\hline 21 & 11 & LABRADOR ICING & $90-180 \mu \mathrm{m}$ \\
\hline 23 & 12 & 102205 & FK \\
\hline 25 & 13 & 102203 & FK \\
\hline 27 & 14 & $\mathrm{H} 22550$ & FK \\
\hline 29 & 15 & 095201 & FK \\
\hline 31 & 16 & OLIGOCLASE & $90-180 \mu \mathrm{m}$ \\
\hline 33 & 17 & ALBITE SWEDEN & $70-180 \mu \mathrm{m}$ \\
\hline 35 & 18 & A4- ANNI & $180-250 \mu \mathrm{m}$ \\
\hline 37 & 19 & 095201 & $\mathrm{FN}$ \\
\hline 39 & 20 & 065414 & FK $180-250 \mu \mathrm{m}$ \\
\hline 41 & 21 & 093071 & FK $90-180 \mathrm{~mm}$ \\
\hline 43 & 22 & 072255 & \\
\hline 45 & 23 & АНO16 & \\
\hline 47 & 24 & 082105 & \\
\hline
\end{tabular}

